[5]. In the latter group, mortality and morbidity are high during infancy because compression of the trachea and right main stem bronchus produces severe respiratory distress within the first year of life. However, asymptomatic pulmonary artery slings are typically diagnosed incidentally in adolescence or adulthood.

Pulmonary artery sling can be repaired in infancy with low operative mortality and excellent long-term patency of the left pulmonary artery, by dividing the left pulmonary artery and implanting it into the main pulmonary artery anterior to the trachea [6]. In contrast to symptomatic patients, the prognosis for asymptomatic patients is excellent and surgical intervention is not indicated.

\author{
E. Martínez-Quintana*, F. Rodríguez-González" and \\ P. Junquera-Rionda" \\ ${ }^{*}$ Cardiology Service, "Radiology Service, Complejo Hospitalario \\ Universitario Insular Materno Infantil, and \#Ophthalmology \\ Service, Hospital Universitario de Gran Canaria Dr. Negrín, Las \\ Palmas de Gran Canaria, Spain.
}

Correspondence: E. Martínez-Quintana, Alcalde Ramírez Bethencourt 10, 3-A 35003, Las Palmas de Gran Canaria, Spain.
E-mail: efrenmartinezquintana@yahoo.es

Statement of Interest: None declared.

\section{REFERENCES}

1 Oster ME, Riehle-Colarusso T, Correa A. An update on cardiovascular malformations in congenital rubella syndrome. Birth Defects Res A Clin Mol Teratol 2010; 88: 1-8.

2 Taylor DM. Maternal rubella and pulmonary arterial stenoses. $\mathrm{Br}$ Med J 1964; 1: 1131.

3 Ellis JG, Kuzman WJ. Pulmonary artery stenosis, a frequent part of the congenital rubella syndrome. Calif Med 1966; 105: 435-439.

4 Davies M, Guest PJ. Developmental abnormalities of the great vessels of the thorax and their embryological basis. Br J Radiol 2003; 76: 491-502.

5 Castañer E, Gallardo X, Rimola J, et al. Congenital and acquired pulmonary artery anomalies in the adult: radiologic overview. Radiographics 2006; 26: 349-371.

6 Backer CL, Idriss FS, Holinger LD, et al. Pulmonary artery sling. Results of surgical repair in infancy. J Thorac Cardiovasc Surg 1992; 103: 683-691.

\title{
Effects of fasudil in patients with high-altitude pulmonary hypertension
}

\section{To the Editors:}

High-altitude pulmonary arterial hypertension (HAPH) is characterised by increased pulmonary vascular resistance (PVR) secondary to hypoxia-induced pulmonary vasoconstriction and vascular remodelling of pulmonary arterioles [1]. Among the potential therapeutic targets is the RhoA/Rho kinases (ROCK) signalling pathway. The GTPase RhoA is a member of the Rho protein family, which regulates cellular functions such as contraction, motility, proliferation and apoptosis, and ROCKs are the best characterised downstream targets for RhoA [2, 3]. Activation of the small GTP-binding protein RhoA and its downstream target ROCK play a significant role in the pathogenesis of pulmonary hypertension (PH). The effectiveness of Rho/ ROCK inhibition has been shown in several murine models of $\mathrm{PH}$ [4-7]. Fasudil, a ROCK inhibitor, is a potent vasodilatator approved in Japan for the treatment of brain vessel vasospasm induced by subarachnoid haemorrhage. It has been shown to reduce PVR in patients with severe idiopathic pulmonary arterial hypertension (PAH) $[8,9]$. The aim of our study was to investigate the potential therapeutic effects of fasudil on pulmonary artery pressure $(P$ pa) in Kyrgyz highlanders with HAPH.

19 patients with $\mathrm{HAPH}$, all permanent residents of the Tien-Shan Mountains (altitude 3,200-3,600 m) were studied. The study was approved by the ethics committee of the National Center of Cardiology and Internal Medicine (Bishkek, Kyrgyzstan) and all subjects gave informed consent. All subjects underwent health screening by history, physical examination, ECG, spirometry, blood pressure measurement and biochemical analysis for liver and kidney functions in order to exclude any pathology that might have potential influence on $\mathrm{HAPH}$.

The effects of fasudil hydrochloride hydrate (Eril; Asahi Kasei Pharma Corp., Tokyo, Japan) and placebo (saline vehicle) on pulmonary haemodynamics were compared in a randomised, double-blind study. Each patient attended our high-altitude hospital at 3,600 $\mathrm{m}$ on two occasions, 1 day apart. On each occasion, echocardiographic studies were performed at baseline and after $30 \mathrm{~min}$ rest. Following this, fasudil or placebo were administered intravenously in a dose of $1 \mathrm{mg} \cdot \mathrm{min}^{-1}$ for the following $30 \mathrm{~min}$ (total dose of fasudil $30 \mathrm{mg}$ ). Echocardiography was started $5 \mathrm{~min}$ before the fasudil or placebo infusion finished. Cardiac frequency $(f \mathrm{C})$, systemic arterial pressure and arterial oxygen saturation $\left(\mathrm{S}_{\mathrm{a}}, \mathrm{O}_{2}\right)$ were monitored continuously during the study (PROPAQ 102; Protocol Systems Inc., Dallas, TX, USA).

Doppler echocardiography was performed using a portable ultrasound system equipped with a $2.5-\mathrm{MHz}$ probe (SpectraMax; SonoSite, Bothell, WA, USA). Systolic $P$ pa was estimated from the systolic pressure gradient between the right ventricle and the right atrium by the peak continuous-wave Doppler velocity of the tricuspid regurgitation jet velocity (TRV) using the modified Bernoulli equation plus right atrial pressure estimated from the inferior vena cava size and collapsibility with respiration. A pulsed Doppler pulmonary blood flow velocity signal was 
TABLE 1 Effects of fasudil on pulmonary artery pressure in Kyrgyz highlanders

\begin{tabular}{|c|c|c|c|c|}
\hline \multirow[t]{2}{*}{ Parameters } & \multicolumn{2}{|c|}{ Placebo } & \multicolumn{2}{|c|}{ Fasudil } \\
\hline & Baseline & $30 \mathrm{~min}$ & Baseline & $30 \mathrm{~min}$ \\
\hline Ppa,sys mmHg & $51.9 \pm 0.7$ & $51.9 \pm 0.8$ & $51.8 \pm 0.7$ & $41.5 \pm 0.6^{\star * *}$ \\
\hline PAAT ms & $0.087 \pm 0.001$ & $0.086 \pm 0.001$ & $0.087 \pm 0.001$ & $0.102 \pm 0.001^{* * \star}$ \\
\hline $\mathrm{CO} \mathrm{L} \cdot \mathrm{min}^{-1}$ & $6.2 \pm 0.2$ & $6.2 \pm 0.3$ & $6.1 \pm 0.3$ & $6.6 \pm 0.2$ \\
\hline \multicolumn{5}{|c|}{ Blood pressure $\mathrm{mmHg}$} \\
\hline Systolic & $125.2 \pm 4.0$ & $125.0 \pm 4.0$ & $125.2 \pm 4.1$ & $122.8 \pm 4.1$ \\
\hline Diastolic & $78.7 \pm 1.5$ & $78.7 \pm 1.6$ & 78.61 .6 & $76.3 \pm 1.5$ \\
\hline fc bpm & $71.3 \pm 2.6$ & $70.8 \pm 2.7$ & $71.4 \pm 2.6$ & $72.0 \pm 2.8$ \\
\hline $\mathrm{Sa}, \mathrm{O}_{2} \%$ & $88.3 \pm 1.1$ & $88.0 \pm 0.9$ & $88.2 \pm 0.7$ & $87.9 \pm 0.6$ \\
\hline
\end{tabular}

Data are presented as mean \pm SEM. Ppa,sys: systolic pulmonary artery pressure; PAAT: pulmonary artery acceleration time; CO: cardiac output; PVR: pulmonary vascular resistance; Pra: right atrial pressure; $f_{C}$ : cardiac frequency; $\mathrm{Sa}_{1} \mathrm{O}_{2}$ : arterial oxygen saturation. ${ }^{* *}: \mathrm{p}<0.001$, fasudil versus placebo.

sampled in the right ventricular outflow tract and a pulmonary artery acceleration time (PAAT) was defined as the time interval from the onset of forward flow in the pulmonary artery to the peak velocity of this flow. Additionally, the velocity-time integral (VTI) of the systolic flow in the right ventricular outflow tract (RVOT) was measured and PVR was calculated using the following formula: PVR $=($ TRV /VTIRVOT $) \times 10+0.16$ [10]. Cardiac output was calculated as a product of the area, VTIRVOT and $f C$. Satisfactory TRV and pulmonary flow velocity signals were obtained in all patients. All measurements were performed by an experienced echocardiographer who was blinded to the study groups. All measurements were validated by another independent observer.

Data are presented as mean \pm SEM. Differences between groups were assessed by ANOVA and Student Newman-Keuls post hoc test for multiple comparisons and a $p$-value $<0.05$ was regarded as significant.

All highlanders had a shortened PAAT, and high systolic $P$ pa and PVR at baseline, consistent with HAPH. Fasudil significantly increased PAAT by $0.015 \pm 0.001 \mathrm{~ms}$ (95\% CI $0.012-0.017 \mathrm{~ms}$, $\mathrm{p}<0.001)$ and decreased systolic $P$ pa by $-10.37 \pm 0.97 \mathrm{mmHg}(95 \%$ CI $-12.34--8.40 \mathrm{mmHg}$, $\mathrm{p}<0.001)$ compared with placebo. Although intravenous infusion of fasudil did not significantly increase cardiac output, it markedly decreased PVR by $-0.39 \pm 0.05$ Wood Units $(95 \%$ CI $-0.49--0.29, p<0.001)$. No changes in systemic arterial blood pressure were noted. $f C$ and $\mathrm{Sa}_{\mathrm{a}} \mathrm{O}_{2}$ were not changed significantly by either fasudil or placebo infusion (table 1). Fasudil was well tolerated; one patient had facial flushing and four patients had feelings of dryness of the mouth.

Our study demonstrates a potent effect of the ROCK inhibitor fasudil on $P$ pa in HAPH. It was well tolerated, with no effect on systemic arterial blood pressure. The data are consistent with the acute effects of fasudil in animal models, which also report on the long-term effects of fasudil administration. Chronic treatment with fasudil effectively reduced $\mathrm{PH}$ and right ventricular hypertrophy, and improved survival in rats with monocrotaline-induced PAH [4-6]. OKA et al. [7] have demonstrated that intravenous fasudil injection was effective in a severe angioproliferative model of PAH. Fasudil reverses monocrotaline-induced $\mathrm{PAH}$ by reducing pulmonary vascular remodelling without affecting normal right ventricular function and may be more effective than bosentan and sildenafil [6]. DAHAL et al. [5] have reported beneficial effects from another ROCK inhibitor, azaindole-1, in two well-established models of $\mathrm{PH}$. Moreover, it significantly inhibited acute hypoxic pulmonary vasoconstriction ex vivo and the proliferation of primary rat pulmonary artery smooth muscle cells in vitro [5]. There are few data in humans. Acute intravenous administration of fasudil at a dose of $1 \mathrm{mg} \cdot \mathrm{min}^{-1}$ for $30 \mathrm{~min}$ effectively reduced PVR in patients with severe PAH $[8,9]$. FUJITA et al. [11] used inhaled fasudil in patients with $\mathrm{PAH}$ and observed that it was as effective as nitric oxide inhalation. Our data indicate that the RhoA/ROCK signalling pathway is involved in the pulmonary vascular response to life at high altitude. A further study of chronic ROCK inhibition is now indicated.

\section{B. Kojonazarov*,\# A. Myrzaakhmatova", T. Sooronbaev", T. Ishizaki ${ }^{+}$and A. Aldashev*}

*Institute of Molecular Biology and Medicine, "National Center of Cardiology and Internal Medicine, Bishkek, Kyrgyzstan, "University of Giessen Lung Center, Giessen, Germany, and ${ }^{+}$Respiratory Division, University Hospital of Fukui, Fukui, Japan.

Correspondence: T. Ishizaki, Respiratory Division, University Hospital of Fukui, Shimoaizuki 23, Matsuoka, Eiheijicho, Fukui Prefecture 910-1104, Japan. E-mail: takeshi@u-fukui.ac.jp

Support Statement: This study was supported by a grant from the Japanese Ministry of Education, Culture, Sport, Science and Technology (No.20406021).

Statement of Interest: None declared. 


\section{REFERENCES}

1 Galie N, Hoeper MM, Humbert M, et al. Guidelines for the diagnosis and treatment of pulmonary hypertension. Eur Respir J 2009; 34: 1219-1263.

2 Somlyo AP, Somlyo AV. Signal transduction by G-proteins, rhokinase and protein phosphatase to smooth muscle and non-muscle myosin II. J Physiol 2000; 522: 177-185.

3 Etienne-Manneville S, Hall A. Rho GTPases in cell biology. Nature 2002; 420: 629-635.

4 Abe K, Shimokawa H, Morikawa K, et al. Long-term treatment with a Rho-kinase inhibitor improves monocrotaline-induced fatal pulmonary hypertension in rats. Circ Res 2004; 94: 385-393.

5 Dahal BK, Kosanovic D, Pamarthi PK, et al. Therapeutic efficacy of azaindole-1 in experimental pulmonary hypertension. Eur Respir J 2010; 36: 808-818.

6 Mouchaers KT, Schalij I, de Boer MA, et al. Fasudil reduces monocrotaline-induced pulmonary arterial hypertension: comparison with bosentan and sildenafil. Eur Respir J 2010; 36: 800-807.
7 Oka M, Homma N, Taraseviciene-Stewart L, et al. Rho kinasemediated vasoconstriction is important in severe occlusive pulmonary arterial hypertension in rats. Circ Res 2007; 100: 923-929.

8 Fukumoto Y, Matoba T, Ito A, et al. Acute vasodilator effects of a Rho-kinase inhibitor, fasudil, in patients with severe pulmonary hypertension. Heart 2005; 91: 391-392.

9 Ishikura K, Yamada N, Ito M, et al. Beneficial acute effects of rhokinase inhibitor in patients with pulmonary arterial hypertension. Circ J 2006; 70: 174-178.

10 Abbas AE, Fortuin FD, Schiller NB, et al. A simple method for noninvasive estimation of pulmonary vascular resistance. $J \mathrm{Am}$ Coll Cardiol 2003; 41: 1021-1027.

11 Fujita H, Fukumoto Y, Saji K, et al. Acute vasodilator effects of inhaled fasudil, a specific Rho-kinase inhibitor, in patients with pulmonary arterial hypertension. Heart Vessels 2010; 25: 144-149.

\section{Nuclear magnetic resonance-based metabolomics of exhaled breath condensate: methodological aspects}

\section{To the Editors:}

Due to the lack of standardised procedures for exhaled breath condensate (EBC), a noninvasive technique for investigating lung inflammatory mediators [1], the between-laboratory comparison of results is difficult. Moreover, different collecting devices have been reported to influence the EBC content [2,3].

The analysis of metabolic profiles ("metabolomics") of EBC using nuclear magnetic resonance (NMR) spectroscopy discriminates between chronic obstructive pulmonary disease (COPD) patients and healthy subjects (HS) [4]; asthmatic children and HS [5]; and patients with stable cystic fibrosis and unstable cystic fibrosis and HS [6].

This approach has recently been questioned as NMR-based metabolomics of EBC collected using a condenser with reusable parts was reported to be affected by cleaning procedures, generating artificial signals that were not related to the endogenous metabolites of the lungs [7].

In this study we assessed the effects of a different cleaning procedure of a reusable-part condenser on EBC metabolomics; the possible time and carry-over effects when the same device is repeatedly used; technique sensitivity; the ability of NMR spectroscopy of EBC to discriminate between COPD patients and HS; and the potential of NMR spectroscopy in identifying selective EBC metabolites.

If the cleaning procedure produces artificial signals in the NMR spectra of EBC, the separation between COPD patients and HS reported previously [4] is certainly surprising, as the residual signals derived from the disinfectant Descogen (Antiseptica chem.-pharm. Produkte GmbH, Pulheim/Brauweiler, Germnay) should have randomly affected both groups. Moreover, using a different reusable-part condenser, CARRARO et al. [5] reported that
NMR-based metabolomics of EBC differentiates asthmatic children from HS with a success rate of $86 \%$ [5].

To verify the influence of the disinfectant on EBC metabolomics, we modified the proposed cleaning procedure [4] by replacing Descogen with Milton (Milton Pharmaceutical UK Ltd, Gloucester, UK), a pure sodium hypochlorite solution. The reusable parts of the EcoScreen condenser (Jaeger, Hoechberg, Germany) were soaked for 15 min with a $3.55 \mathrm{mM}$ Milton solution (according to manufacturer guidelines), and then flushed for $15 \mathrm{~min}$ with deionised distilled water. ${ }^{1} \mathrm{H}-\mathrm{NMR}$ spectra were recorded at $600 \mathrm{MHz}(14.1 \mathrm{~T})$ on a Bruker Avance spectrometer (Bruker BioSpin $\mathrm{GmbH}$, Rheinstetten, Germany) equipped with a 5-mm CPTCI CryoProbe ${ }^{\mathrm{TM}}$ (Bruker BioSpin GmbH).

Figure 1a reports the NMR spectrum of the Milton solution. Except for the reference $0.1 \mathrm{mM}$ sodium 3-trimethylsilyl $\left[2,2,3,3-{ }^{2} \mathrm{H}_{4}\right]$ propionate (TSP) signal at $0.0 \mathrm{ppm}$, no other signals were present in the spectrum. The absence of signals (implying the absence of contaminant) was confirmed in the vertical expansion of the spectrum (fig. 1b). To exclude the presence of contaminants in the deionised water used for rinsing the collection set-up, we acquired the spectrum of the soaking water after rinsing for $5 \mathrm{~min}$ (fig. 1c). No peak was observed, indicating that the disinfection and the rinsing steps of the EBC collection set-up do not affect the EBC spectra. These data exclude the fact that cleaning procedure based on Milton solution affects NMR spectroscopy of EBC, indicating that the observed profiles of EBC metabolites are not artificial signals but represent endogenous metabolites.

When a reusable-part condenser (EcoScreen) was repeatedly used [8], the measurement of several EBC biomarkers detected no carry-over effects from previous sampling periods. If carry-over effects were present, the metabolites would display an "average 